\section{Questions les plus fréquentes sur le TarMed}

Je prévois, comme médecin interniste, d'ouvrir un cabinet médical en 2001 et de me consacrer essentiellement à la psychothérapie (j'ai une formation appropriée de plusieurs années dans ce domaine). Après l'introduction du TarMed, pourrai-je sans problème facturer les psychothérapies aux caisses-maladie et, si oui, selon quel tarif?

H. K. $\dot{a} E$.

Cela ne sera malheureusement pas possible. Les médecins qui commenceront à facturer, à titre individuel, selon les tarifs fondés sur le TarMed après l'entrée en vigueur de ceux-ci ne pourront pas être mis au bénéfice des dispositions transitoires et, donc, de la garantie des droits acquis.

Je suis praticien en médecine générale et dirige depuis 1994 mon propre institut de physiothérapie, avec une physiothérapeute diplômée comme employée. Comment se fera la facturation des prestations de physiothérapie dans le TarMed et la nouvelle tarification est-elle à peu près comparable à l'ancienne?

J. B. $\dot{a} D$.

Le TarMed ne comprend pas de prestations de physiothérapie. Ces dernières doivent être facturées selon le tarif de physiothérapie. Demeurent réservées, les prestations de rééducation et de physiothérapie spécialisée qui sont en cours de traitement.

Actuellement, le désir de certains groupes de spécialistes se précise de combiner des valeurs intrinsèques élevées avec des productivités aussi basses que possible. Dans ce contexte, on peut se demander à combien s'élève la valeur intrinsèque du temps d'attente et du temps d'inoccupation du cabinet.

Prenons l'exemple d'une prestation à valeur intrinsèque élevée qui dure en moyenne $40 \mathrm{~min}$, avec un temps d'inoccupation de 20 min. Le taux de productivité horaire est donc de 0,66. Dans cet exemple facture-t-on l'heure entière selon la valeur intrinsèque élevée ou uniquement la prestation effective de 40 min?

\section{U. H. $\dot{a} T$.}

Idée très intéressante que celle qui voudrait que le temps d'attente soit facturé selon une valeur intrinsèque de 12 . Il en résulterait une situation de gain à $\mathrm{n}$ dimensions ne pouvant être définie que de façon très vague. Le litige concernant la tarification appropriée des prestations serait balayé d'un coup, de même que les questions de responsabilité civile (plus personne ne faisant quoi que ce soit), les détracteurs de la technologie médicale seraient contents parce que la médecine aurait atteint le nirvana du rien absolu. Les problèmes seraient en fait réservés aux assureurs et politiciens, ou peut être pas non plus, car ils sont habitués à ce que leur attente soit très bien rémunérée. Soyons sérieux, la réalité est tout autre: il y a, sur ce point, égalité de tous devant la loi (à part pour quelques exceptions qualifiées) et l'attente est assortie pour tous d'une valeur intrinsèque de 5 ; en outre, cette attente n'est pas non plus illimitée, car la productivité, là aussi, ne saurait admettre qu'elle s'étende jusqu'à l'infini.

Est-il correct que, dans la version alpha 2.2 du TarMed, la ponction biopsie hépatique percutanée (19.1340) soit facturée avec 309.25 pts pour la prestation technique (PT) plus 104.73 pts pour la prestation médicale (PM), alors que pour la biopsie de transplantation rénale du néphrologue FMH, presque aussi difficile, on peut facturer au mieux 30.2850, c.-à-d. 44.32 pts pour la PT plus 49.44 pts pour la PM? Négligence ou erreur intentionnelle?

A. B. $\dot{a} A$.

Cette question sera examinée lors de la révision 2000, car ce n'est en effet pas logique. Qui pourrait avoir une telle intention?

Le TarMed est subdivisé en prestations médicales (PM) et prestations techniques (PT).

Lors des négociations tarifaires cantonales prévues, seule la PT, qui comprend les coûts d'infrastructure, devrait être négociée. La PM étant la prestation médicale intellectuelle, elle devrait donc être identique dans toutes les régions du pays, également pour l'AI, la CNA et les caisses-maladie. Etes-vous d'accord avec ce point de vue?

Est-il nécessaire, à votre avis, qu'on applique la même procédure dans tous les cantons pour les négociations tarifaires, autrement dit que l'on opère des comparaisons de factures entre l'ancien et le nouveau tarif, etc.?

E. S. $\dot{a} D$.

Ad 1: Je partage votre point de vue. Cela dit, un certain nombre de personnes ayant un rôle déterminant ne sont pas du même avis.

Ad 2: En principe oui. Mais les sociétés cantonales de médecine sont libres de s'associer en régions.

Je suis généraliste FMH depuis près de 20 ans. Pendant mon assistanat, $j$ 'ai également fait une année de psychiatrie et, par la suite, j'ai régulièrement participé aux groupes Balint et, enfin, j'ai suivi une formation de 5 ans en psychothérapie centrée sur la personne (env. 1500 heures). Cela fait donc des années que je fournis des prestations de psychothérapie (env. le $50 \%$ de mon activité professionnelle) et que je les facture selon le tarif des caisses-maladie. 
a) Pourrai-je continuer à travailler comme psychothérapeute médical sous le régime du nouveau TarMed et à facturer sans restriction selon le tarif pour la psychothérapie?

b) Aurai-je encore le droit de déléguer un traitement à une psychothérapeute qualifiée?

A. W. $\dot{a} Z$

En vertu du principe des droits acquis, oui.

Je suis psychiatre et psychothérapeute et travaille environ 42 heures par semaine à mon cabinet. Environ 10 à $15 \%$ de mes heures de thérapie sont perdues parce que les patients oublient leur rendez-vous ou le renvoient à court terme. Comme, chez moi, il n'y a jamais personne en salle d'attente (les séances durent entre 30 et 50 minutes), je ne peux pas compenser ces séances perdues. Cette particularité du cabinet psychiatrique est-elle prise en compte quelque part?

T. H. $\dot{a} B$.

Oui, il est tenu compte de cet aspect dans la productivité des différents secteurs (la psychiatrie en est un). Le taux de productivité a été établi d'après l'enquête sur le temps de travail, à laquelle ont également participé des psychiatres, et il se situe à $85 \%$ dans le secteur psychiatrique.

En tant que spécialiste FMH en orthopédie, je me pose les questions suivantes:

1. Que signifie une valeur intrinsèque FMH 8?

2. Je suis spécialiste dans ce domaine depuis 1998; quelle est donc "ma valeur intrinsèque»?

3. Celle-ci augmenterait-elle si je gardais un poste de formation encore quelques années?

4. Est-ce que 10 années d'assistanat, par exemple, ont la même valeur que 10 années d'activité en tant que chef de clinique dans un domaine chirurgical tel que l'orthopédie, avec toutes les interventions pratiquées? Cela ne joue-t-il aucun rôle dans la détermination de la valeur intrinsèque?

Honnêtement, tout cela est loin d'être facile à comprendre...

D. T. $\dot{a} P$.
La valeur intrinsèque quantitative est en corrélation avec la durée de formation postgraduée nécessaire pour pouvoir fournir une prestation sous sa propre responsabilité et donc pour pouvoir la facturer; dans près de $90 \%$ des cas, elle correspond aux cursus de formation pour le titre de spécialiste correspondant. Il s'agit en fait d'un SIMPLE FACTEUR DE CALCUL qui est SANS IMPORTANCE pour l'utilisateur. Autrement dit: celui qui peut facturer des prestations à valeur intrinsèque quantitative 12 , ne peut pas facturer toutes les prestations et encore moins réévaluer les prestations, p. ex. la consultation, d'un facteur 12 en ce qui le concerne.

Pour vous, ce qui compte c'est la valeur intrinsèque qualitative; celle-ci indique si, en vertu de votre titre de spécialiste ou - le cas échéant - de qualifications complémentaires, vous pouvez facturer la prestation.

Comme interniste avec 8 ans de formation en médecine interne et dans ses sous-spécialités, je suis mis au même niveau qu'un généraliste, dans ce système tarifaire. Pourquoi n'y a-t-il aucune différence entre un spécialiste FMH en médecine interne et un spécialiste FMH en médecine générale quant au droit de facturer des prestations spécifiques ( $p$. ex. mesure de la pression sur 24 h, ergométrie, ECG Holter, test fonctionnel endocrinologique, etc.)? Malgré une formation plus différenciée, je suis considéré au même titre que le simple médecin de premier recours, sur le plan tarifaire.

W. C. $\dot{a} E$.

Les sociétés suisses de médecine générale, de médecine interne et de pédiatrie ont participé dans une large mesure ensemble aux négociations, en tant que sociétés de médecins de premier recours; c'est ce qui explique que les valeurs intrinsèques qualitatives sont en grande partie identiques pour les trois sociétés et leurs titres. 


\section{Facharztprüfung zur Erlangung des Facharzttitels FMH für Orthopädische Chirurgie}

Aufgrund des Weiterbildungsprogrammes, welches am 1. Januar 2000 in Kraft gesetzt wurde, ist die Teilnahme an der Facharztprüfung Voraussetzung für die Erlangung des Facharzttitels FMH für Orthopädische Chirurgie. Es empfiehlt sich, die Facharztprüfung frühestens im letzten Jahr der reglementarischen Weiterbildung abzulegen (Art. 23 WB0).

Basisexamen: Wer die Weiterbildung zum Facharzt FMH für Orthopädische Chirurgie am 1. Januar 2000 bereits begonnen hat, ist von der Teilnahme am chirurgischen Basisexamen befreit.
1. Teil (schriftliche Prüfung)

Samstag, 26. August 2000

2. Teil (praktische/mündliche Prüfung)

Samstag, 25. November 2000

Prüfungsgebühr: Die SGO erhebt einen Unkostenbeitrag von Fr. 600.-.

Anmeldung: Die Anmeldeformulare können bezogen werden bei: Sekretariat der SG0, Postfach, 3000 Bern 25, Tel. 03133296 10, Fax 03133298 79, E-mail: bbscongress@swissonline.ch

Anmeldefrist: 31. Juli 2000

\section{Examen de spécialiste pour l'obtention du titre FMH en chirurgie orthopédique}

Conformément au programme de formation postgraduée, entré en vigueur le $1^{\mathrm{er}}$ janvier 2000, la participation à l'examen de spécialiste est une condition requise pour le titre de spécialiste FMH en chirurgie orthopédique. Il est recommandé aux candidats de passer l'examen de spécialiste au plus tôt durant la dernière année de leur formation postgraduée réglementaire (art. 23 RFP).

Examen de base: Tout candidat ayant commencé sa formation postgraduée pour le titre de spécialiste FMH en chirurgie orthopédique avant le $1^{\text {er }}$ janvier 2000 sera dispensé de l'examen chirurgical de base. $1^{\text {re }}$ partie (examen écrit)

Samedi, le 26 août 2000

$2^{\text {ème }}$ partie (examen pratique/oral)

Samedi, le 25 novembre 2000

Taxe d'examen: La SSO prélève une participation aux frais de Fr. 600.-.

Inscription: Les formulaires d'inscription peuvent être obtenus auprès du Secrétariat SSO, case postale, 3000 Berne 25, tél. 03133296 10, fax 03133298 79, e-mail: bbscongress@swissonline.ch

Délai d'inscription: le 31 juillet 2000 


\section{Facharztprüfung zur Erlangung des Facharzttitels FMH für Anästhesiologie}

Erster Teil (schriftliche Prüfung) Samstag, 7. Oktober 2000, in Bern

Zweiter Teil (mündliche Prüfung) Samstag, 27. Januar 2001, in Bern

Prüfungsgebühr: Fr. 600.-

Anmeldung:

Die Anmeldeformulare können bezogen werden bei: Sekretariat der SGAR, Frau B. Bühlmann, Postfach, 3000 Bern 25, Tel. 031332 34 33, Fax 0313329879.

Anmeldefrist: 31. Mai 2000

\section{Examen de spécialiste en vue de l'obtention du titre FMH en anesthésiologie}

Première partie (examen écrit)

Samedi, 7 octobre 2000, à Berne

Deuxième partie (examen oral)

Samedi, 27 janvier 2001, à Berne

Taxe d'examen: Fr. 600.-

Inscription:

Les formulaires d'inscription peuvent être obtenus auprès du Secrétariat de la SSAR, Mme B. Bühlmann, Case postale, 3000 Berne 25, tél. 03133234 33, fax 0313329879.

Délai d'inscription: le 31 mai 2000 